\title{
FURTHER DEVELOPMENT OF THE HYBRID MODEL FOR POLYCRYSTAL DEFORMATION
}

\author{
J. V. FERNANDES ${ }^{\dagger} \uparrow$ and M. F. VIEIRA \\ ${ }^{1}$ Departamento de Engenharia Mecânica-CEMUC, Faculdade de Ciências e Tecnologia da \\ Universidade de Coimbra, Polo II da Universidade de Coimbra, Pinhal de Marrocos, P-3030 Coimbra, \\ Portugal and ${ }^{2}$ Departamento de Engenharia Metalúrgica e de Materiais, GMM/IMAT, Faculdade de \\ Engenharia da Universidade do Porto, Rua dos Bragas, P-4050-123 Porto, Portugal
}

(Received 18 December 1998; accepted 8 December 1999)

\begin{abstract}
The Kocks-Mecking hybrid model for polycrystal deformation has been developed further, in order to obtain an easy definition of polycrystalline behaviour, from knowledge of the single crystal stressstrain curves. The hybrid model is a one-parameter theory that considers the flow stress governed by total dislocation density. This is the addition of the statistically stored dislocations, independent of the grain size, and the geometrical dislocations, necessary to accommodate the deformation, and thus dependent on the grain size (as proposed by Ashby). The condition for the onset of necking is used to determine the parameters of the equation of the model. The analytical description of the resulting flow law was used to analyse the tensile behaviour of copper sheets, with five different grain sizes, which were deformed at three different strain rates. (C) 2000 Acta Metallurgica Inc. Published by Elsevier Science Ltd. All rights reserved.
\end{abstract}

Keywords: Mechanical properties (constitutive equations); Dislocations (theory); Copper

\section{INTRODUCTION}

The plastic deformation of metals has been extensively studied over the last decades: see, for example, Refs [1-3]. The formulation of the most recent models describing the flow stress has been developed by considering complex microstructures present in cold-deformed metals $[4,5]$. In this way, several microstructural elements are taken into account in these new physically based theories, as, for example, the dislocation densities inside the cells, the cell size, the cell structure and misorientation. Such models have to incorporate certain elements of empiricism in order to cover phenomena, which nevertheless needs further research.

Single-parameter models (see Refs [6-10], for example) are still extensively used in the modelling of metal plasticity. These models consider that the flow stress is given by an equation of the type $\tau=\tau(\rho, \dot{\gamma}, T)$, where $\dot{\gamma}$ and $T$ are the strain rate and temperature, respectively, and $\rho$ is the total dislocation density, which represents the current structure. This equation needs to be complemented by another that describes the structural evolution with strain, at a given strain rate and temperature: $\mathrm{d} \rho / \mathrm{d} \gamma=\mathrm{f}(\rho, \dot{\gamma}, T)$. The evolution rate of the dislo-

$\dagger$ To whom all correspondence should be addressed. cation density depends on hardening and on dynamic recovery components: the former is of an athermal nature and the latter is influenced by thermal activation. For low dislocation density, at the beginning of deformation, the hardening rate depends on the athermal nature of dislocation-dislocation interaction, being independent of the strain rate and temperature. When plastic deformation $\gamma$ increases, the parameter $\rho$ evolves to a saturation value $\rho_{\mathrm{s}}$ due to the dynamic recovery mechanisms and the flow stress also tends toward saturation value $\tau_{\mathrm{s}}$. The usual formulation of the one-parameter model leads to a Voce type equation to represent flow behaviour [11, 12].

During tension of polycrystals, the macroscopic flow stress $\sigma$ and strain $\varepsilon$ can be calculated from $\tau$ and $\gamma$ as follows: $\sigma=M \gamma, \varepsilon=\gamma / M$, where $M$ is a parameter independent of the grain size (the Taylor factor, for example). This parameter accounts for the passage of the stress and strain in active slip systems to macroscopic stress and strain [13-15]. Besides, in the case of polycrystals the evolution of dislocation density with strain depends on the grain size [16-23]. At a given strain value, when the grain size decreases, the density of dislocation increases. Also, the number of active slip systems can increase [24]. Both effects promote the accommodation of local plastic deformation, between adjacent grains. 
In fact, the accommodation process imposes multiple slip inside the grains and the total dislocation density increases quickly, mainly at the beginning of deformation, depending on the grain size. After Ashby [25], the rate of dislocation storage during polycrystal deformation depends on two terms. One is equivalent to the stored dislocation term, in the case of monocrystal. The other term deals with socalled geometrical dislocations, which are necessary in the accommodation of plastic deformation between the grains of the polycrystal. This allows formulation of the hybrid model for polycrystal deformation. Basically, this model considers two production terms of dislocations, as mentioned above, and an annihilation term.

This work focuses on the effects of the grain size on the parameters of the hybrid model for polycrystal deformation. The hybrid model is developed by introducing a parameter that characterises the relationship between the two production terms. The influence of the value of this parameter on the evolution of the density of dislocations with deformation, and on work-hardening behaviour, is considered. We deduced and discussed the possibility of using the condition of the onset of necking as an easy determination for some parameters of the hybrid model. An example of an experimental study is shown: polycrystalline copper samples with five different grain sizes were deformed in tension, using three different strain rates.

\section{ANALYTICAL DESCRIPTION OF THE FLOW
LAW}

\subsection{Hardening and dynamic recovery compounds of microstructural evolution}

The current stress $\tau$ can be decomposed into two additive contributions, related to lattice friction and to the interaction between dislocations:

$$
\tau=\tau_{0}+\alpha \mu b \sqrt{\rho}
$$

where $b$ is the magnitude of the Burgers vector, $\mu$ the shear modulus, $\rho$ the dislocation density and $\alpha$ a numerical factor, which is mainly dependent on the material and characterises the strength of dislocation-dislocation interaction $(\alpha=0.5-1.0)$. In f.c.c. metals, the value of $\tau_{0}$ can be neglected, in order to simplify analytical development.

The rate of stress variation, with the strain $\theta=\mathrm{d} \tau / \mathrm{d} \gamma$, can be deduced from the above equation, as follows (see Refs [6-10], for example):

$$
\theta=\frac{\mathrm{d} \tau}{\mathrm{d} \gamma}=\frac{\alpha \mu b}{2 \sqrt{\rho}} \frac{\mathrm{d} \rho}{\mathrm{d} \gamma} .
$$

Stress $\tau$ depends on the evolution of dislocation density with deformation. This evolution is the result of competition between the production rate and the annihilation rate of dislocations (see Refs
[6-10, 26-30], for example):

$$
\frac{\mathrm{d} \rho}{\mathrm{d} \gamma}=\left(\frac{\mathrm{d} \rho}{\mathrm{d} \gamma}\right)^{+}-\left(\frac{\mathrm{d} \rho}{\mathrm{d} \gamma}\right)^{-}
$$

Both terms in this equation can be deduced from the physical mechanisms of deformation. In the case of monocrystals, the production term is associated with athermal storage (independent of the temperature and strain rate) of moving dislocations that become immobilised, after travelling the mean free path $\lambda$. This term is proportional to the average spacing between dislocations $\sqrt{\rho}(\lambda=\beta / \sqrt{\rho}, \beta$ being the constant of proportionality- $\beta \approx 100$ ) and it is possible to define a constant of proportionality $k_{1}$ between $(\mathrm{d} \rho / \mathrm{d} \gamma)^{+}$and $\sqrt{\rho}$ such that $k_{1}=1 / b \beta$.

The annihilation rate, associated to dynamic recovery (stage III), is assumed to be linear in $\rho(k$ being the constant of proportionality). As the annihilation event occurs when dislocations of opposite signs pass at a distance $R$ apart, $k$ can be considered as being $k \propto 2 R / b[8,9,26]$. The single parameter theory describes the thermally activated softening term $(\mathrm{d} \rho / \mathrm{d} \gamma)^{-}$as a function of the strain rate $\dot{\gamma}$ and the absolute temperature at a given structure $S$, which can be represented by the dislocation density $\rho:(\mathrm{d} \rho / \mathrm{d} \gamma)^{-}=f(S, \dot{\gamma}, T)=f(\rho, \dot{\gamma}, T)$. So, it is possible to form the following equations:

$$
\left(\frac{\mathrm{d} \rho}{\mathrm{d} \gamma}\right)^{+}=\frac{1}{b \lambda}=\frac{\sqrt{\rho}}{b \beta}=k_{1} \sqrt{\rho}\left(\frac{\mathrm{d} \rho}{\mathrm{d} \gamma}\right)^{-}=k \rho .
$$

In the case of polycrystals, the dislocation density at a constant strain value depends on the grain size. The dislocation model, proposed by Ashby [25], allows for the separation of the strength contribution to those that are independent and dependent of the grain size. Thus, the rate of stored dislocation is the addition of two terms:

$$
\left(\frac{\mathrm{d} \rho}{\mathrm{d} \gamma}\right)^{+}=\left(\frac{\mathrm{d} \rho}{\mathrm{d} \gamma}\right)^{\mathrm{S}}+\left(\frac{\mathrm{d} \rho}{\mathrm{d} \gamma}\right)^{\mathrm{G}}
$$

The first term concerns so-called statistical dislocations and is equivalent to the stored dislocations term in the case of monocrystal [first part of equation (3)]. The second term concerns geometrical dislocations in order to accommodate the plastic deformation between grains of polycrystals:

$$
\left(\frac{\mathrm{d} \rho}{\mathrm{d} \gamma}\right)^{\mathrm{G}}=\frac{1}{b \lambda^{\mathrm{G}}}=\frac{c}{b D}=k_{2}
$$

where $\lambda^{\mathrm{G}}$ is the mean free path of the geometrical dislocations, which is constant for each grain size $D$ and proportional to it (through the constant $c$, being $c \approx 1$ for copper).

Finally, the evolution of the dislocation density with deformation can be written as follows: 


$$
\frac{\mathrm{d} \rho}{\mathrm{d} \gamma}=k_{1} \sqrt{\rho}+k_{2}-k \rho .
$$

According to this equation, the dislocation density evolves towards a steady state value $\rho_{\text {sat }}$ for which $(\mathrm{d} \rho / \mathrm{d} \gamma)=0$, such as:

$$
\begin{aligned}
\sqrt{\rho_{\mathrm{sat}}} & =\frac{k_{1}}{k} \frac{\left(1+\sqrt{1+4 \frac{k k_{2}}{k_{1}^{2}}}\right)}{2} \\
& =\frac{k_{1}}{k} \frac{(1+\sqrt{1+4 x})}{2}
\end{aligned}
$$

$x$ being a value where:

$$
x=\frac{k k_{2}}{k_{1}^{2}} .
$$

The term $x / k=k_{2} / k_{1}^{2}$ is not dependent on dislocation annihilation and thus it independently characterises the relative production of geometrical dislocations. For monocrystals, there is no production of geometrical dislocations, $k_{2}=0$, and consequently, $x=0$. In coarse-grain size polycrystals, the geometrical dislocations play a minor role in deformation and $k_{2} \rightarrow 0$ (so, $x \rightarrow 0$ ).

With regard to the annihilation parameter $k$, we account for the possibility that the value of $k$ depends on the grain size. In fact, the annihilation rate can be influenced by the arrangement of dislocations (see Ref. [26], for example). This one is a function of the grain size as has been studied previously [16-23]. In this context, $k$ will be called $k^{\mathrm{V}}$ for deformation microstructures typical of monocrystals. For coarse-grain size polycrystals, it is expected that the value of the annihilation parameter $k$ is close to $k^{\mathrm{V}}$.

Therefore, equation (8) can now be written as follows:

$$
\sqrt{\rho_{\mathrm{sat}}}=\sqrt{\rho_{\mathrm{sat}}^{\mathrm{V}}} \frac{(1+\sqrt{1+4 x})}{2} .
$$

In this equation, $\sqrt{\rho_{\text {sat }}^{\mathrm{V}}}=k_{1} / k^{\mathrm{V}}$ represents the saturation dislocation density, in the case of monocrystals or coarse-grain size polycrystals (as above mentioned), i.e. in the absence of representative density of geometrical dislocations.

\subsection{The relationship between geometrical and} statistical dislocation densities

Equations (4) and (6) allow us to represent both the statistical and geometrical dislocation densities in the grain, as a function of the strain, as follows (neglecting dislocation density at the beginning of deformation, in order to simplify the equation):

$$
\log \rho^{\mathrm{S}}=2 \log k_{1}+2 \log \gamma-2 \log 2
$$

$$
\log \rho^{\mathrm{G}}=2 \log k_{1}+\log \gamma+\log \frac{x}{k} .
$$

It is now possible to determine the evolution of the ratio between the cumulated production of geometrical and statistical dislocation densities during deformation by an equation of the form:

$$
\log \left(\frac{\rho^{\mathrm{G}}}{\rho^{\mathrm{S}}}\right)=-\log \gamma+\log \left(4 \frac{x}{k}\right) .
$$

So, the knowledge of the value $(x / k)=\left(k_{2} / k_{1}^{2}\right)$ for a given grain size, which is independent of dislo-
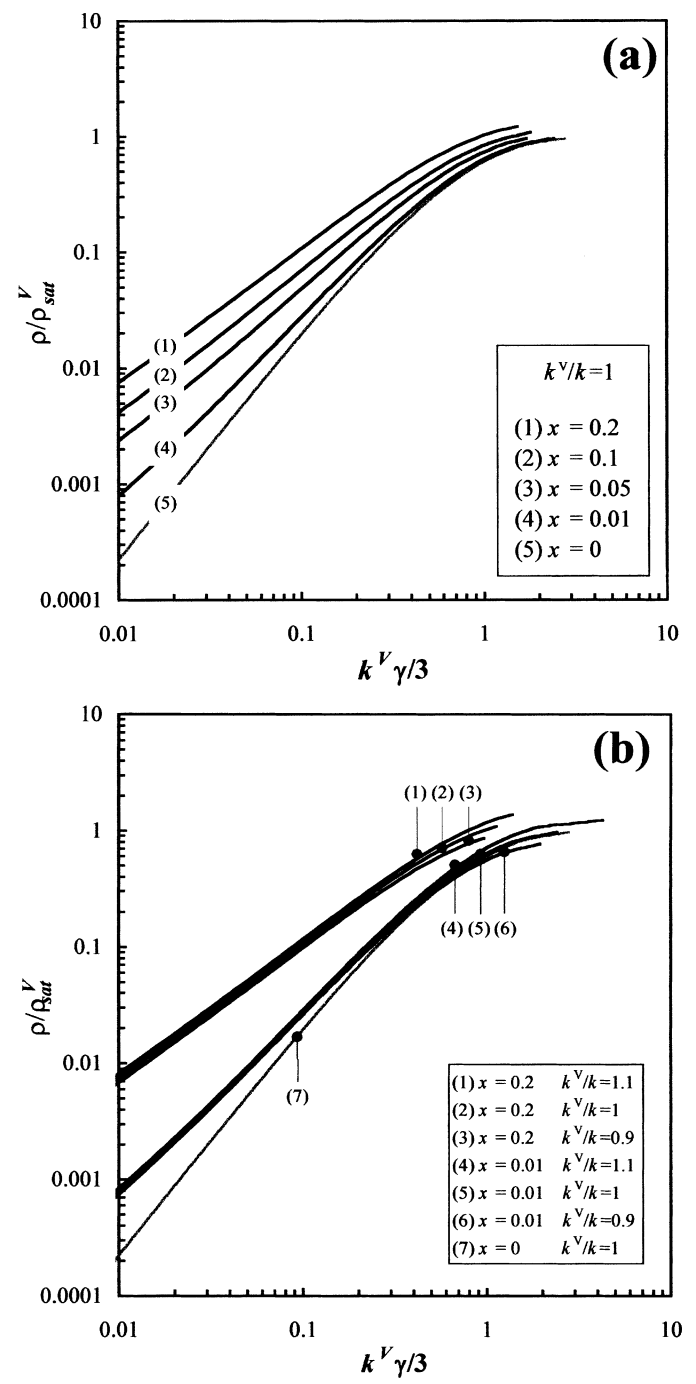

Fig. 1. Evolution of the ratio $\left(\rho / \rho_{\text {sat }}^{\mathrm{V}}\right)$, between the dislocation density and the respective saturation value in the absence of geometrical dislocations, as a function of $\left(k^{\mathrm{V}} \gamma / 3\right)$, for several $x$ values: (a) different $x$ values without change in the ratio $\left(k^{\mathrm{v}} / k=1\right)$; (b) two $x$ values, different to zero; for each one, three different $\left(k^{\mathrm{V}} / k\right)$ values are considered $\left(k^{\mathrm{v}} / k=0.9,1.0\right.$ and 1.1). Note: the value of $\left(k \gamma^{\mathrm{V}} / 3\right)$ is close to the shear strain $\gamma$ for copper; in this case, the $k$ values are close to 3 (Table 2 shows that $M k$ is between 8.50 and 9.94 , the Taylor factor being $M=3.06$ for a material without texture), as discussed in the text. 
cation annihilation as mentioned above, allows for the determination of the relative evolution of geometrical and statistical dislocation densities.

Through the integration of equation (7) it is possible to describe the evolution of the ratio $\rho / \rho_{\text {sat }}^{\mathrm{V}}$ (between the dislocation density $\rho$ and respective saturation value $\rho_{\text {sat }}^{\mathrm{V}}$, where geometrical dislocations are absent) as a function of the shear strain $\gamma$ in the grain, by the equation (neglecting the initial dislocation density, in order to simplify the equation):

$$
\begin{gathered}
\left\{\frac{1}{\sqrt{1+4 x}} \ln \left[\frac{\sqrt{\frac{\rho}{\rho_{\text {sat }}^{\mathrm{V}}}} \frac{k}{k^{\mathrm{V}}}(1+\sqrt{1+4 x})+2 x}{\sqrt{\frac{\rho}{\rho_{\text {sat }}^{\mathrm{V}}}} \frac{k}{k^{\mathrm{V}}}(1-\sqrt{1+4 x})+2 x}\right]\right. \\
-\ln \left[-\left(\sqrt{\frac{\rho}{\rho_{\text {sat }}^{\mathrm{V}}}} \frac{k}{k^{\mathrm{V}}} \frac{1}{\sqrt{x}}\right)^{2}\right. \\
\left.\left.+\sqrt{\frac{\rho}{\rho_{\text {sat }}^{\mathrm{V}}}} \frac{k}{k^{\mathrm{V}}} \frac{1}{x}+1\right]\right\}\left(\frac{k^{\mathrm{V}}}{k}\right)=k^{\mathrm{V} \gamma} .
\end{gathered}
$$

Consequently, the stress evolves towards a steady state value as follows:

$$
\sigma_{\mathrm{sat}}=M \alpha \mu b \frac{k_{1}}{k} \frac{(1+\sqrt{1+4 x})}{2} .
$$

The integration of equation (15) allows us to write:

$$
\begin{aligned}
& \varepsilon-\varepsilon_{\mathrm{c}}= \\
& \left(\frac{1}{M k}\right)\left\{\frac{1}{\sqrt{1+4 x}} \ln \left[\frac{\left[-\frac{\sigma}{\sigma_{\mathrm{sat}}}(1+\sqrt{1+4 x})+1-\sqrt{1+4 x}\right]\left[-\frac{\sigma_{\mathrm{c}}}{\sigma_{\mathrm{sat}}}(1+\sqrt{1+4 x})+1+\sqrt{1+4 x}\right]}{\left[-\frac{\sigma}{\sigma_{\mathrm{sat}}}(1+\sqrt{1+4 x})+1+\sqrt{1+4 x}\right]\left[-\frac{\sigma_{\mathrm{c}}}{\sigma_{\mathrm{sat}}}(1+\sqrt{1+4 x})+1-\sqrt{1+4 x}\right]}\right]\right. \\
& \left.-\ln \left[\frac{-\left(\frac{\sigma}{\sigma_{\mathrm{sat}}} \frac{1+\sqrt{1+4 x}}{2}\right)^{2}+\frac{\sigma}{\sigma_{\mathrm{sat}}} \frac{1+\sqrt{1+4 x}}{2}+x}{-\left(\frac{\sigma_{\mathrm{c}}}{\sigma_{\mathrm{sat}}} \frac{1+\sqrt{1+4 x}}{2}\right)^{2}+\frac{\sigma_{\mathrm{c}}}{\sigma_{\mathrm{sat}}} \frac{1+\sqrt{1+4 x}}{2}+x}\right]\right\}
\end{aligned}
$$

Examples of the results of this equation are shown in Figs 1(a) and (b). It is possible to see in this figure that the geometrical dislocation density shows its importance long after the respective density becomes much less than statistical dislocation. Considering that $k$ and consequently the value of the ratio $k^{\mathrm{V}} / k$ depends on the grain size, the curves in Fig. 1(b) cross each other and the corresponding stress evolves similarly with deformation. This can be an additional reason for the curves crossing, which is sometimes observed when the grain size is changed. A common explanation considers texture effects [31, 32]. where $\varepsilon_{\mathrm{c}}$ and $\sigma_{\mathrm{c}}$ are the integration constants, corresponding to a point on the tensile curve.

When the geometrical dislocations can be neglected, the above equation becomes the Voce equation $(x=0)$ as follows:

$$
\sigma=\sigma_{\text {sat }}^{\mathrm{V}}-\left(\sigma_{\text {sat }}^{\mathrm{V}}-\sigma_{\mathrm{c}}\right) \exp \left[-\frac{M k^{\mathrm{V}}}{2}\left(\varepsilon-\varepsilon_{\mathrm{c}}\right)\right] .
$$

In this equation, the value $k^{\mathrm{V}}$ can be different from those of $k$, as in the case of equation (14), which depends on the structure and consequently on the 

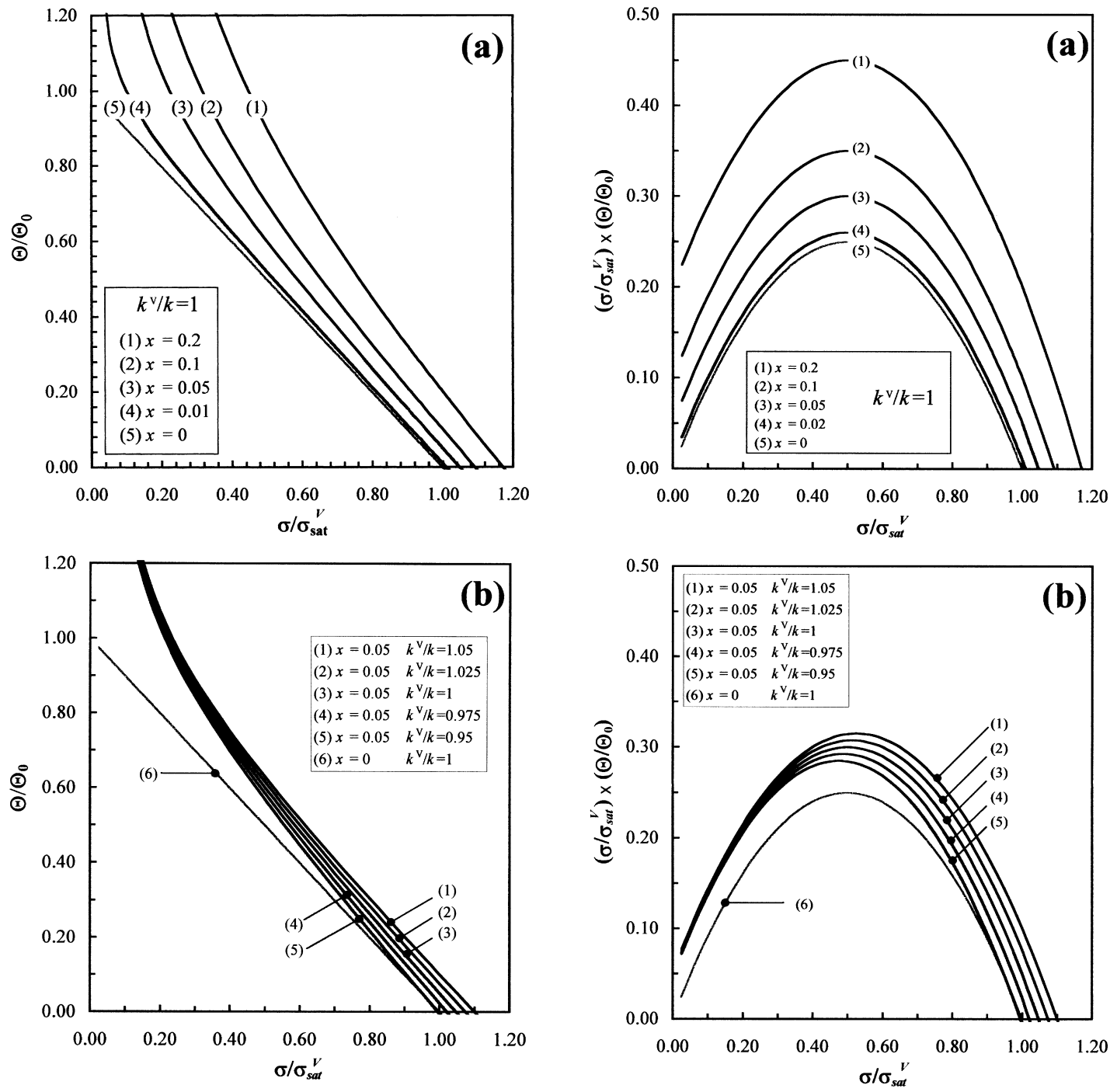

Fig. 2. $\Theta / \Theta_{0}$ vs $\sigma / \sigma_{\text {sta }}^{\mathrm{v}}$ diagram for: (a) $k^{\mathrm{v}} / k=1$, with different $x$ values; (b) $x=0.05$, with different $k^{\mathrm{V}} / k$ values.

grain size. In fact, the rate of dynamic recovery may be dependent on the type of dislocation structure $[5,26,33,34]$, which in turn depends on the grain size [24]. Other parameters such as temperature and strain rate also influence its value.

The linear relationship in the $\Theta(=\mathrm{d} \sigma / \mathrm{d} \varepsilon)=f(\sigma)$ diagram, which characterises the Voce equation, can be obtained as follows:

$$
\Theta=\Theta_{0}\left(1-\frac{\sigma}{\sigma_{\text {sat }}^{\mathrm{V}}}\right)
$$

where $\Theta_{0}=M^{2} \theta_{\mathrm{II}}$, the quantity $\theta_{\mathrm{II}}$ being related to the stage II hardening of monocrystals. It is recognised that $\theta_{\mathrm{II}}$ and consequently $\Theta_{0}$ are independent of the strain rate and depend on the temperature through the temperature dependence of $\mu$ [6-10]. An important feature of the dislocation storage is its athermal character:

Fig. 3. $\left(\sigma / \sigma_{\text {sat }}^{\mathrm{V}}\right)\left(\Theta / \Theta_{0}\right)$ vs $\sigma / \sigma_{\text {sat }}^{\mathrm{v}}$ diagram for the same cases as in Fig. 2: (a) $k \mathrm{v} / k=1$, with different $x$ values; (b) $x=$ 0.05 , with different $k^{\mathrm{V}} / k$ values.

$$
\Theta_{0}=M^{2} \theta_{\mathrm{II}}=M^{2} \frac{\alpha \mu b}{2} k_{1}
$$

It is now possible to express the saturation stress $\sigma_{\text {sat }}^{\mathrm{V}}$ for coarse-grained material, which obeys the Voce equation $(x=0)$, as a function of $\Theta_{0}$ and $M k^{\mathrm{V}}$ :

$$
\sigma_{\mathrm{sat}}^{\mathrm{V}}=\frac{2 \Theta_{0}}{M k^{\mathrm{V}}}=M \alpha \mu b \frac{k_{1}}{k^{\mathrm{V}}}
$$

In the general case of fine-grained materials, the saturation stress $\sigma_{\text {sat }}$ [equation (13)] can be expressed as a function of $\Theta_{0}, M k$ and $x$ as follows:

$$
\begin{aligned}
\sigma_{\mathrm{sat}} & =\frac{\Theta_{0}(1+\sqrt{1+4 x})}{M k} \\
& =\sigma_{\mathrm{sat}}^{\mathrm{V}} \frac{k^{\mathrm{V}}}{k} \frac{(1+\sqrt{1+4 x})}{2} .
\end{aligned}
$$


The value of $M k$ can be determined from equation (18):

$$
\frac{1}{M k}=\frac{\sigma_{\mathrm{sat}}^{\mathrm{V}}}{2 \Theta_{0}} \frac{k^{\mathrm{V}}}{k} .
$$

It is now possible to write equation (17) as follows: from zero, the origin of these curves is vertically displaced, and the curves for small grain sizes can cross the large grain size curve, for low $k^{\mathrm{v}} / k$ values.

\subsection{The condition for the onset of necking}

In the knowledge that at the beginning of necking

$$
\begin{aligned}
\varepsilon-\varepsilon_{\mathrm{c}}= & \left(\frac{\sigma_{\text {sat }}^{\mathrm{V}}}{2 \Theta_{0}} \frac{k^{\mathrm{V}}}{k}\right)\left\{\frac { 1 } { \sqrt { 1 + 4 x } } \operatorname { l n } \left[\frac{\left[-2 \frac{\sigma}{\sigma_{\text {sat }}^{\mathrm{V}}} \frac{k}{k^{\mathrm{V}}}+1-\sqrt{1+4 x}\right]\left[-2 \frac{\sigma_{\mathrm{c}}}{\sigma_{\text {sat }}^{\mathrm{V}}} \frac{k}{k^{\mathrm{V}}}+1+\sqrt{1+4 x}\right]}{\left.\left[-2 \frac{\sigma}{\sigma_{\text {sat }}^{\mathrm{V}}} \frac{k}{k^{\mathrm{V}}}+1+\sqrt{1+4 x}\right]\left[-2 \frac{\sigma_{\mathrm{c}}}{\sigma_{\text {sat }}^{\mathrm{V}}} \frac{k}{k^{\mathrm{V}}}+1-\sqrt{1+4 x}\right]\right]}\right.\right. \\
& \left.-\ln \left[\frac{-\left(\frac{\sigma}{\sigma_{\text {sat }}^{\mathrm{V}}} \frac{k}{k^{\mathrm{V}}}\right)^{2}+\frac{\sigma}{\sigma_{\text {sat }}^{\mathrm{V}}} \frac{k}{k^{\mathrm{V}}}+x}{-\left(\frac{\sigma_{\mathrm{c}}}{\sigma_{\mathrm{sat}}^{\mathrm{V}}} \frac{k}{k^{\mathrm{V}}}\right)^{2}+\frac{\sigma_{\mathrm{c}}}{\sigma_{\text {sat }}^{\mathrm{V}}} \frac{k}{k^{\mathrm{V}}}+x}\right]\right\} .
\end{aligned}
$$

Also, from equations (20) and (22) and by utilising equation (15), it is possible to write:

$$
\begin{gathered}
\frac{\Theta}{\Theta_{0}}=1+\frac{2 x}{1+\sqrt{1+4 x}} \frac{1}{\frac{\sigma}{\sigma_{\text {sat }}}}-\frac{\sigma}{\sigma_{\text {sat }}} \frac{1+\sqrt{1+4 x}}{2} \\
\frac{\Theta}{\Theta_{0}}=1+\frac{x}{\frac{\sigma}{\sigma_{\text {sat }}^{\mathrm{V}}}} \frac{k^{\mathrm{V}}}{k}-\frac{\sigma}{\sigma_{\text {sat }}^{\mathrm{V}}} \frac{k}{k^{\mathrm{V}}} .
\end{gathered}
$$

It is now possible to determine the evolution of the work-hardening rate as a function of the stress. This is shown in Figs 2(a) and (b) for several cases of the values of $x$ and $k^{\mathrm{v}} / k$. When $x$ increases, the stress value, for which work hardening attains $\Theta_{0}$, also increases. This effect is seen up to saturation stress: whatever the stress value, the $\Theta$ values are greater for larger $x$ values. For the same $x$ value, the saturation stress is determined by the relative value $k^{\mathrm{v}} / k$.

An alternative way to analyse work-hardening behaviour is by using a diagram type $\sigma \Theta=f(\sigma)$ as follows:

$$
\begin{aligned}
& \frac{\sigma}{\sigma_{\text {sat }}} \frac{\Theta}{\Theta_{0}}= \frac{2 x}{1+\sqrt{1+4 x}}+\frac{\sigma}{\sigma_{\text {sat }}} \\
&-\left(\frac{\sigma}{\sigma_{\text {sat }}}\right)^{2} \frac{1+\sqrt{1+4 x}}{2} \\
& \frac{\sigma}{\sigma_{\text {sat }}^{\mathrm{V}}} \frac{\Theta}{\Theta_{0}}=x \frac{k^{\mathrm{V}}}{k}+\frac{\sigma}{\sigma_{\text {sat }}^{\mathrm{V}}}-\left(\frac{\sigma}{\sigma_{\text {sat }}^{\mathrm{V}}}\right)^{2} \frac{k}{k^{\mathrm{V}}} .
\end{aligned}
$$

In Figs 3(a) and (b), the results corresponding to Figs 2(a) and (b) are shown. For an $x$ value that differs the following condition can be verified:

$$
\left\{\left(\frac{1}{\sigma} \Theta\right)=1\right\}_{\text {necking }}
$$

it is possible to determine the value $\sigma_{\text {sat }}^{\mathrm{V}}$ as:

$$
\sigma_{\mathrm{sat}}^{\mathrm{V}}=\sigma_{\mathrm{n}}^{\mathrm{V}} \frac{M k^{\mathrm{V}}+2}{M k^{\mathrm{V}}}
$$

where $\sigma_{\mathrm{n}}^{\mathrm{V}}$ is the stress at the instability point corresponding to the maximum load. If we consider that the constants of equation (18) represent the stress and strain at this point, the Voce equation can now be written in a specific formulation, as follows:

$$
\sigma=\sigma_{\mathrm{n}}^{\mathrm{V}}\left\{\frac{M k^{\mathrm{V}}+2}{M k^{\mathrm{V}}}-\frac{2}{M k^{\mathrm{V}}} \exp \left[-\frac{M k^{\mathrm{V}}}{2}\left(\varepsilon-\varepsilon_{\mathrm{n}}^{\mathrm{V}}\right)\right]\right\}
$$

This is an equation, with a physical basis, to describe the mechanical behaviour of a material with large grain size, for which it is necessary to know the values of $\sigma_{\mathrm{n}}^{\mathrm{V}}, \varepsilon_{\mathrm{n}}^{\mathrm{V}}$ and $M k^{\mathrm{V}}$. The values of $\sigma_{\mathrm{n}}^{\mathrm{V}}$ and $\varepsilon_{\mathrm{n}}^{\mathrm{V}}$ can be directly obtained from the experimental tensile curves. As the texture evolution can be neglected during deformation for values up to approximately 0.35 , the value of $M k^{\mathrm{V}}$ can be considered as being constant during a tensile test. Besides, the value of $M k^{\mathrm{V}}$ can be related to $\varepsilon_{\mathrm{n}}^{\mathrm{V}}$ from equation (25):

$$
\varepsilon_{\mathrm{n}}^{\mathrm{V}}=\frac{2}{M k^{\mathrm{V}}} \ln \left(\frac{M k^{\mathrm{V}}+2}{2}\right) .
$$

Therefore, knowledge of the value of $\sigma_{\mathrm{n}}^{\mathrm{V}}$ and $\varepsilon_{\mathrm{n}}^{\mathrm{V}}$ allows us to determine the constants of equation (18) and consequently to plot the curve given by 
this equation, which is suitable for f.c.c. materials of coarse-grained size.

In the case of fine-grained materials, the geometrical dislocations cannot be neglected and it is not possible to find an easy method to determine the parameters of equation (17). However, equations (14), (15) and (21) allow us to obtain the following relationship:

$$
\begin{aligned}
\frac{2}{M k}= & x\left[\frac{2 \sigma_{\text {sat }}}{\sigma_{\mathrm{n}}(1+\sqrt{1+4 x})}\right]^{2} \\
& +\frac{2 \sigma_{\text {sat }}}{\sigma_{\mathrm{n}}(1+\sqrt{1+4 x})}-1 \\
& =x\left(\frac{\sigma_{\text {sat }}^{\mathrm{V}}}{\sigma_{\mathrm{n}}}\right)^{2}\left(\frac{k^{\mathrm{V}}}{k}\right)^{2}+\frac{\sigma_{\text {sat }}^{\mathrm{V}}}{\sigma_{\mathrm{n}}} \frac{k^{\mathrm{V}}}{k}-1 .
\end{aligned}
$$

Also, from equation (15) and taking into account equations (20) and (22), it is possible to write:

$$
\sigma\left(\Theta=\Theta_{0}\right)=\frac{2 \sigma_{\text {sat }}}{1+\sqrt{1+4 x}} \sqrt{x}=\sigma_{\text {sat }}^{\mathrm{V}} \frac{k^{\mathrm{V}}}{k} \sqrt{x} .
$$

So, knowing the values of $\Theta_{0}$ and $\sigma_{\text {sat }}^{\mathrm{V}}$ obtained from the results of coarse-grained material (or alternatively the knowledge of monocrystal behaviour, through $\theta_{0}$ and $\tau_{\text {sat }}^{\mathrm{V}}$, and the $M$ factor) and plotting the $\Theta(\mathrm{d} \sigma / \mathrm{d} \varepsilon)=f(\sigma)$ diagram of the tensile curve for a fine-grained material, it is possible to determine, by using the above two equations (31) and (32), the corresponding values of $\sqrt{x}$ and $k^{\mathrm{V}} / k$, as follows:

$$
\sqrt{x}=\frac{\frac{\sigma\left(\Theta=\Theta_{0}\right)}{\sigma_{\mathrm{n}}}-\frac{\sigma\left(\Theta=\Theta_{0}\right)}{2 \Theta_{0}}}{1-\left(\frac{\sigma\left(\Theta=\Theta_{0}\right)}{\sigma_{\text {sat }}^{\mathrm{V}}}\right)^{2}}
$$

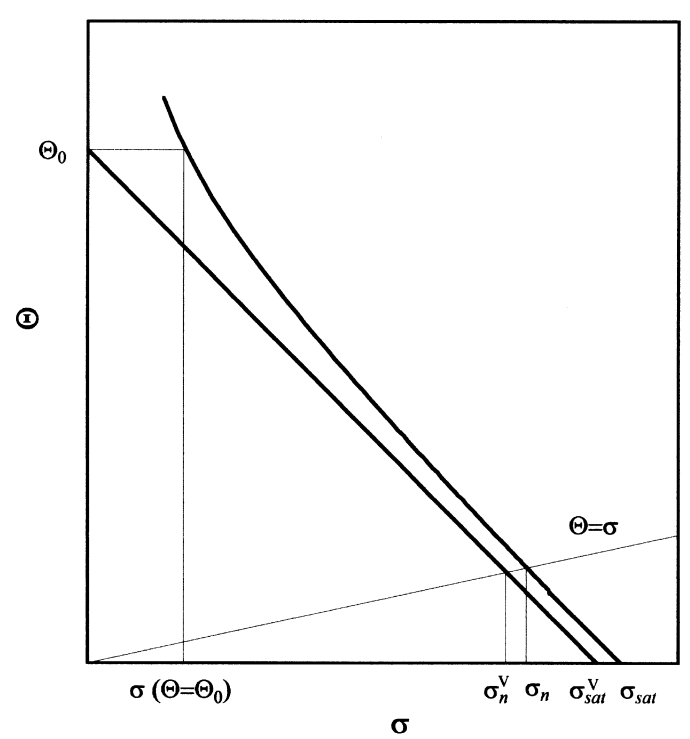

Fig. 4. $\Theta$ vs $\sigma$ diagram representing parameters of equations (33) and (34), such as: $\sigma\left(\Theta=\Theta_{0}\right), \sigma_{\mathrm{n}}^{\mathrm{V}}, \sigma_{\mathrm{n}} \cdot \sigma_{\text {sat }}^{\mathrm{V}}, \sigma_{\text {sat }}$.

$$
\frac{k^{\mathrm{V}}}{k}=\frac{\sigma\left(\Theta=\Theta_{0}\right)}{\sigma_{\text {sat }}^{\mathrm{V}}} \frac{1}{\sqrt{x}} .
$$

In Fig. 4 the parameters used in these equations are represented. This is an easy way to determine the importance of the geometrical dislocations, when the behaviour of a coarse-grained material is known. Another way is to fit the experimental tensile curve through equation (17).

In conclusion, the behaviour of a coarse-grained material can be deduced from the values of $\sigma_{\mathrm{n}}^{\mathrm{V}}$ and $\varepsilon_{\mathrm{n}}^{\mathrm{V}}$ obtained at the maximum load in tension, in particular the values of $M k^{\mathrm{V}}$ and $\sigma_{\text {sat }}^{\mathrm{V}}$ [equations (28), (30) and also using equation (21) to determine $\Theta_{0}$ ]. For other grain sizes (less than approximately $100 \mu \mathrm{m})$, the values of $x$ and $k^{\mathrm{V}} / k$ can be determined from equations (33) and (34). This is by
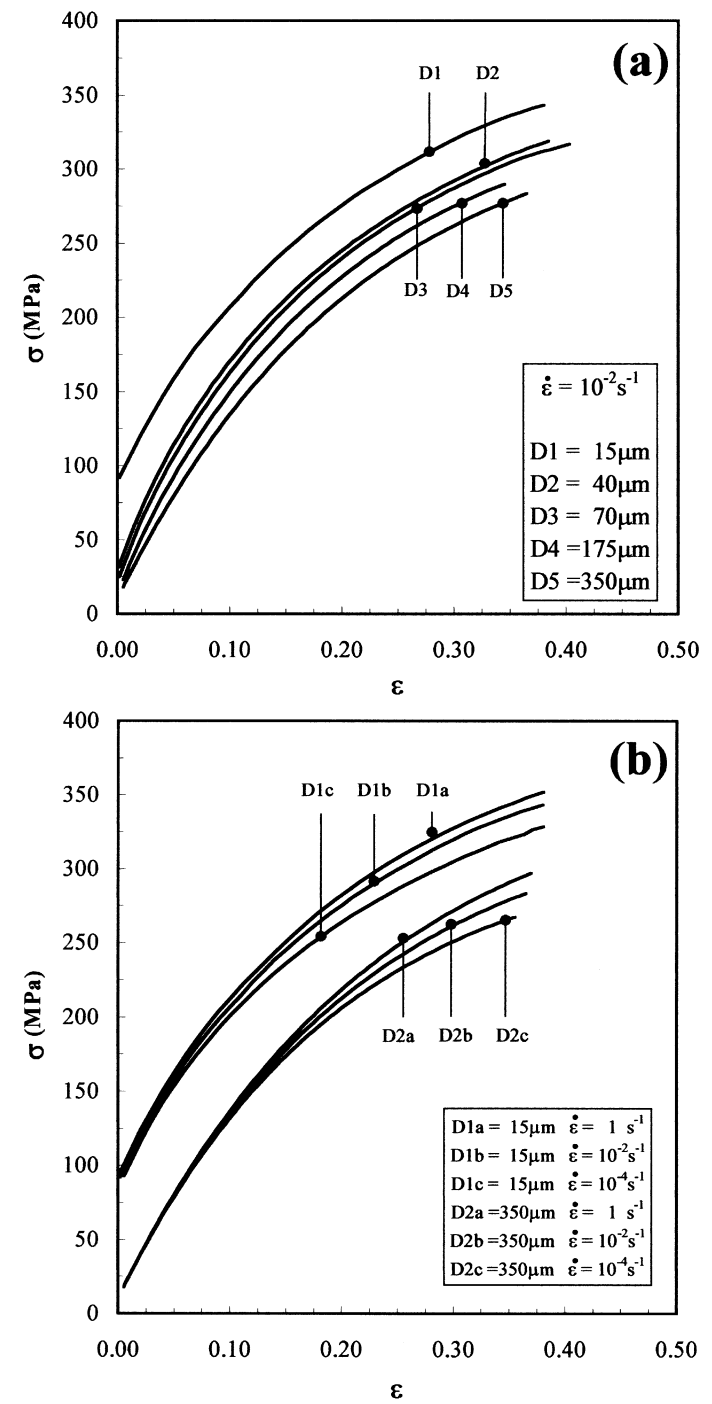

Fig. 5. Examples of true stress $\sigma$-true strain $\varepsilon$ curves for: (a) all the grain sizes studied (15, 40, 70, 175 and $350 \mu \mathrm{m})$, at a strain rate $\dot{\varepsilon}=10^{-2} / \mathrm{s}$; (b) two grain sizes $(15$ and $350 \mu \mathrm{m})$ and the three strain rates studied $\left(\dot{\varepsilon}=10^{-4}, 10^{-2}\right.$ and $1 / \mathrm{s})$. 
tracing the tensile curve for the corresponding grain size and knowing the mechanical behaviour of the same material of coarse grain size, or even the behaviour of the monocrystal.

\section{GRAIN SIZE EFFECT ON THE WORK- HARDENING BEHAVIOUR OF COPPER}

\subsection{Experiments}

Oxygen-free high purity copper $(99.95 \% \quad \mathrm{Cu})$ sheet, $5 \mathrm{~mm}$ thick, previously cold-rolled and annealed, was used in this research. The grains were equiaxed with $15 \mu \mathrm{m}$ grain size. The sheet was subsequently annealed at different temperatures, in order to induce secondary recrystallisation. Four grain sizes were obtained, depending on the temperature, namely $40,70,175$ and $350 \mu \mathrm{m}$ for 400 , 500,650 and $800^{\circ} \mathrm{C}$, respectively, for an annealing time of about $1.5 \mathrm{~h}$. Thermal processing was carried
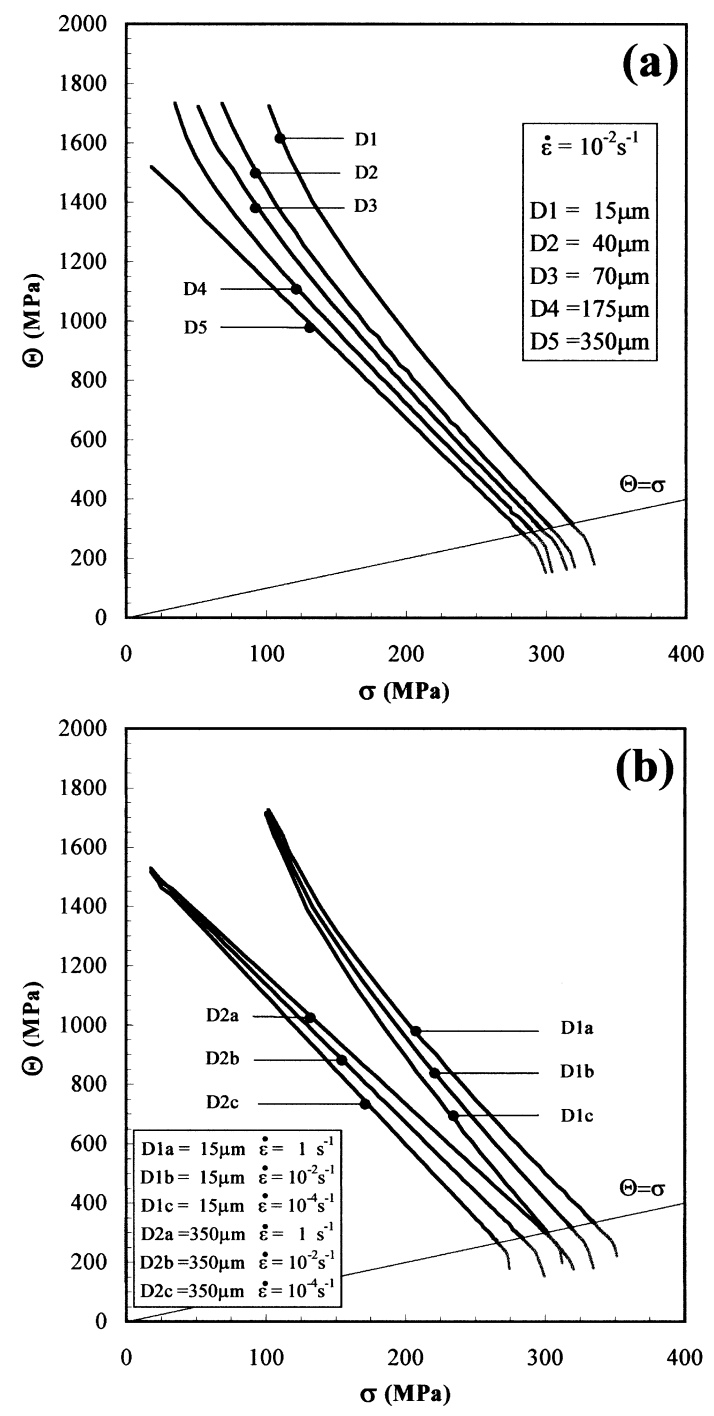

Fig. 6. As Fig. 5, but for the evolution of the work-hardening ratio $\Theta$ as a function of the stress $\sigma$. out on tensile specimens in a $10^{-6}$ Torr vacuum, in order to avoid any hardening during subsequent mechanical operations.

Different texture measurements were performed for the samples of the smallest and the largest grain size, in order to check the influence of subsequent annealing. For every case a weak rolling texture was found. The quantitative comparison of pole figures is rather difficult, owing to the different size of grains compared with the spot size. From these measurements, it must nevertheless be concluded that the same crystallographic orientations are present in both cases and that no differing orientations appear. This result, which seems to be valid for the range of grain sizes studied in this work, is consistent with previous results on copper [24].

The tensile tests were performed along the transverse direction with respect to the rolling direction on ISO 50 samples at room temperature. Three initial von Mises strain rates, close to $10^{-4}, 10^{-2}$ and $1 / \mathrm{s}$, were used. A $50-\mathrm{mm}$ strain gauge was used in order to measure the extension. A microcomputer was interfaced with the tensile test machine and the signals from load and extension were converted and stored through computer software, in the form of true stress $\sigma$-true strain $\varepsilon$. For further processing of these data, computer software was developed, to allow outputs of $\sigma=f(\varepsilon)$ and $\Theta(=\mathrm{d} \sigma / \mathrm{d} \varepsilon)=f(\sigma)$.

\subsection{Results}

Examples of true stress $\sigma$-true strain $\varepsilon$ curves are shown in Fig. 5(a). In this figure it is possible to compare the effects of the grain size on the level of the curves: the smaller the grain, the greater the stress at each strain. Figure 5(b) shows examples of tensile curves at different strain rates.

Figure 6(a) shows the evolution of the workhardening ratio $\Theta$ as a function of the stress $\sigma$. At very low stress, the work hardening is higher as the grain size decreases, for a given strain rate. At greater stress values, higher hardening is still observed for the smaller grain sizes. When changing the strain rate, at a given grain size, the lines start at the same point, and they become slightly further apart for higher stress [Fig. 6(b)].

\subsection{Discussion}

As was previously discussed [24], the influence of geometrical dislocations on polycrystal behaviour drops drastically for copper grain sizes, which are larger than approximately $100 \mu \mathrm{m}$. The present stress-strain curves confirm these results. In fact, the $\Theta=f(\sigma)$ curves for $350 \mu \mathrm{m}$ grain size are quite linear and tend to cross the vertical axes at a finite value $\Theta_{0}$, typical when geometrical dislocations are not considered. So, equations (15) and (16) can be 
Table 1. Results for the case of large grain size $(350 \mu \mathrm{m})$, for all the strain rates studied $\left(\dot{\varepsilon}=10^{-4}, 10^{-2}\right.$ and $\left.1 / \mathrm{s}\right)$, showing the experimental stress $\left(\sigma_{\mathrm{n}}^{\mathrm{V}}\right)$ and strain $\left(\varepsilon_{\mathrm{n}}^{\mathrm{V}}\right)$ values at necking and the parameters of the Voce equation $M k^{\mathrm{V}}, \sigma_{\text {sat }}^{\mathrm{V}}$ and $\Theta_{0}$

\begin{tabular}{|c|c|c|c|c|c|c|c|c|}
\hline $\begin{array}{l}\text { Grain size } \\
(\mu \mathrm{m})\end{array}$ & $\begin{array}{l}\text { Strain rate } \\
\qquad\left(\mathrm{s}^{-1}\right)\end{array}$ & $\begin{array}{c}\sigma_{\mathrm{n}} \\
(\mathrm{MPa})\end{array}$ & $\varepsilon_{\mathrm{n}}$ & $\begin{array}{c}M k^{\mathrm{V}} \text { [equation } \\
(18)]\end{array}$ & $\begin{array}{c}\sigma_{\text {sat }}^{\mathrm{V}} \text { [equation } \\
(18) \text { ] } \\
(\mathrm{MPa})\end{array}$ & $\begin{array}{c}M k^{\mathrm{V}} \text { [equation } \\
(30)]\end{array}$ & $\begin{array}{c}\sigma_{\text {sat }}^{\mathrm{V}} \text { [equation } \\
(28) \text { ] } \\
(\mathrm{MPa})\end{array}$ & $\begin{array}{c}\Theta_{0} \\
(\mathrm{MPa})\end{array}$ \\
\hline 350 & $\begin{array}{r}10^{-4} \\
10^{-2} \\
1\end{array}$ & $\begin{array}{l}266 \\
285 \\
302\end{array}$ & $\begin{array}{l}0.360 \\
0.374 \\
0.387\end{array}$ & $\begin{array}{r}10.01 \\
9.28 \\
8.65\end{array}$ & $\begin{array}{l}320 \\
345 \\
370\end{array}$ & $\begin{array}{l}9.93 \\
9.24 \\
8.64\end{array}$ & $\begin{array}{l}320 \\
347 \\
372\end{array}$ & 1600 \\
\hline
\end{tabular}

used to describe the behaviour of larger grain size $(350 \mu \mathrm{m})$ copper sheets.

For the three strain rates, the values of $M k^{\mathrm{V}}$ and $\sigma_{\text {sat }}^{\mathrm{V}}$ were determined using the results of $350 \mu \mathrm{m}$ grain size. For that, equation (18) was fitted to the experimental results $\sigma=f(\varepsilon)$. Equation (21) allowed us to determine $\Theta_{0}$. This value and the value of $\sigma_{\text {sat }}^{\mathrm{V}}$ were used in equation (19) to compare them with experimental results $\Theta=f(\sigma)$. A good fitting was confirmed for all cases.

Similar results for $M k^{\mathrm{V}}$ and $\sigma_{\text {sat }}^{\mathrm{V}}$ were obtained using equations (30) and (28) (deduced from the condition of the onset of necking) and the experimental values of $\sigma_{\mathrm{n}}$ and $\varepsilon_{\mathrm{n}}$. As shown in Table 1, the values are quite similar, which means that for a large grain size, the complete tensile curve can be deduced from the knowledge of $\sigma_{\mathrm{n}}$ and $\varepsilon_{\mathrm{n}}$ and by using equation (29).

The value of $\Theta_{0}$ is approximately the same, whatever the case of strain rate, which agrees with its independence of the strain rate (and temperature). This value is equal to $1600 \mathrm{MPa}$, similar to the results previously mentioned by other authors $[6,8]$. The values of $\sigma_{\text {sat }}^{\mathrm{V}}$, and consequently $M k^{\mathrm{V}}$, depend on the strain rate. This study is not within the objectives of the present work, but it is possible to observe that this agrees with what can be expected $[5,28,33,34]: \sigma_{\text {sat }}^{\mathrm{V}}$ and $\dot{\varepsilon}$ are quite linear in a logarithmic representation.
For smaller grain sizes, the behaviour cannot be described by the Voce equation and so the equations of the hybrid model must be utilised. In the present case, the values of $x$ and $M k$ were obtained by fitting equation (24) to the experimental curves and using the values of $\Theta_{0}$ and $\sigma_{\text {sat }}^{\mathrm{V}}$ mentioned in Table 1. In Fig. 7 some examples of the comparison between experimental and fitted curves are shown. The values of $x$ and $M k$ obtained by this fitting are also shown in Table 2. A good approximation of the $x$ values is obtained by using equation (33) (deduced from the condition of the onset of necking), when the values of $\sigma\left(\Theta=\Theta_{0}\right)$ and the coarse-grain size behaviour $\left(\Theta_{0}\right.$ and $\left.\sigma_{\text {sat }}^{\mathrm{V}}\right)$ are known. The value of $\sigma\left(\Theta=\Theta_{0}\right)$ can be determined from the curves $\Theta=f(\sigma)$ (Fig. 6) or alternatively as schematically shown in Fig. 8. The results obtained in this way are also shown in Table 2.

To our knowledge there are no written results that concern the influence of the grain size on the annihilation rate of dislocation, through the value of $k$. This value can be determined by taking $M$ as a constant. The present results show that, in this approach, $k$ (or more precisely $M k$ ) is only slightly influenced by the grain size, when compared with the influence of the strain rate on the range $10^{-4}$ to $1 / \mathrm{s}$. This is probably due to a similar internal structure of the cell boundaries, which must be of the same type [24]. Moreover, existing differences in the

Table 2. Results for the case of smaller grain sizes $(15,40,70$ and $175 \mu \mathrm{m})$, for all the strain rates studied $\left(\dot{\varepsilon}=10^{-4}, 10^{-2}\right.$ and $\left.1 / \mathrm{s}\right)$, showing: the experimental stress $\left(\sigma_{\mathrm{n}}\right)$ and strain $\left(\varepsilon_{\mathrm{n}}\right)$ values at necking and the other parameters of equations (24), (25) and (26) such as $x$ and $k^{\mathrm{v}} / k$. The values of $\sigma\left(\Theta=\Theta_{0}\right), \sigma_{\text {sat }}$ and $M k$ are also shown

\begin{tabular}{|c|c|c|c|c|c|c|c|c|c|}
\hline Grain size $(\mu \mathrm{m})$ & Strain rate $\left(\mathrm{s}^{-1}\right)$ & $\sigma_{\mathrm{n}}(\mathrm{MPa})$ & $\varepsilon_{\mathrm{n}}$ & $\sigma\left(\Theta=\Theta_{0}\right)(\mathrm{MPa})$ & $x^{\mathrm{a}}$ & $x^{\mathrm{b}}$ & $x / k^{\mathrm{c}}$ & $\sigma_{\text {sat }}(\mathrm{MPa})$ & $M k$ \\
\hline \multirow[t]{3}{*}{15} & $10^{-4}$ & 324 & 0.361 & 106 & 0.106 & 0.109 & 0.032 & 357 & 9.81 \\
\hline & $10^{-2}$ & 349 & 0.390 & 109 & 0.096 & 0.093 & 0.032 & 383 & 9.10 \\
\hline & 1 & 352 & 0.393 & 112 & 0.088 & 0.097 & 0.031 & 407 & 8.50 \\
\hline \multirow[t]{3}{*}{40} & $10^{-4}$ & 305 & 0.392 & 78 & 0.059 & 0.061 & 0.018 & 341 & 9.92 \\
\hline & $10^{-2}$ & 326 & 0.395 & 81 & 0.055 & 0.056 & 0.018 & 366 & 9.19 \\
\hline & 1 & 336 & 0.381 & 84 & 0.050 & 0.055 & 0.018 & 393 & 8.54 \\
\hline \multirow[t]{3}{*}{70} & $10^{-4}$ & 295 & 0.388 & 64 & 0.039 & 0.042 & 0.012 & 335 & 9.92 \\
\hline & $10^{-2}$ & 321 & 0.405 & 66 & 0.036 & 0.037 & 0.012 & 360 & 9.21 \\
\hline & 1 & 325 & 0.395 & 69 & 0.034 & 0.039 & 0.012 & 387 & 8.55 \\
\hline \multirow[t]{3}{*}{175} & $10^{-4}$ & 277 & 0.364 & 41 & 0.016 & 0.018 & 0.005 & 327 & 9.94 \\
\hline & $10^{-2}$ & 300 & 0.383 & 44 & 0.016 & 0.018 & 0.005 & 353 & 9.21 \\
\hline & 1 & 314 & 0.387 & 45 & 0.015 & 0.017 & 0.005 & 378 & 8.59 \\
\hline
\end{tabular}

${ }^{a}$ Values deduced from equation (33).

${ }^{\mathrm{b}}$ Values corresponding to the best fit of the analytical curves, deduced from equation (24), to the experimental curves.

${ }^{\mathrm{c}}$ Values deduced considering $M=3.06$. 
$k$ value can be hidden by changes in the $M$ parameter value. Nevertheless, the grain growth annealing treatment can slightly change this parameter, which will reflect on the value of the annihilation parameter. However, it is not the purpose of this paper to discuss the influence of the type of dislocation microstructure on the annihilation rate.

The values of $x / k$ increase with the decreasing grain size and they do not depend on the strain rate (Table 2). This is an expected result, as $x / k$ represents in some way a measurement of the geometrical dislocation density, which, for a given material, is solely dependent on the grain size, as follows:

(a)

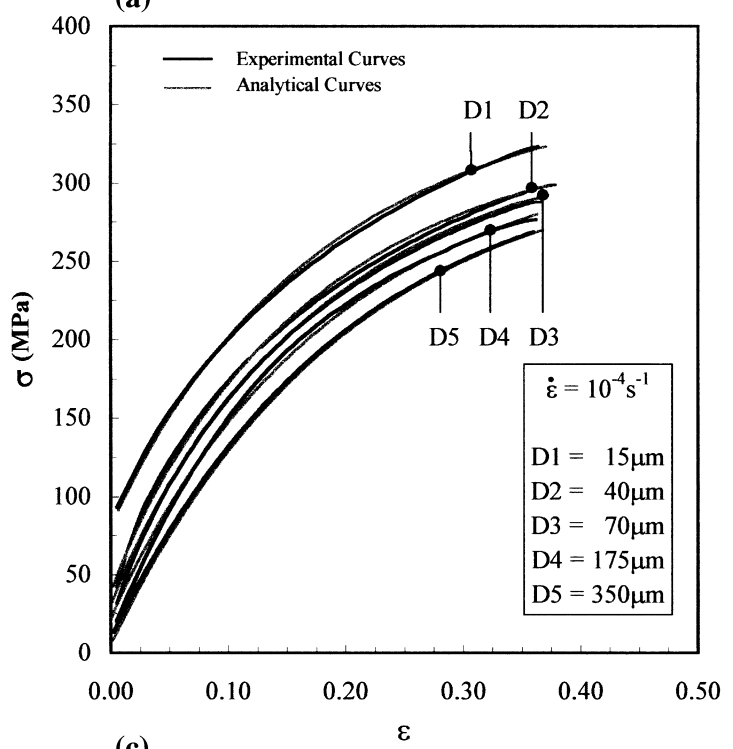

(c)

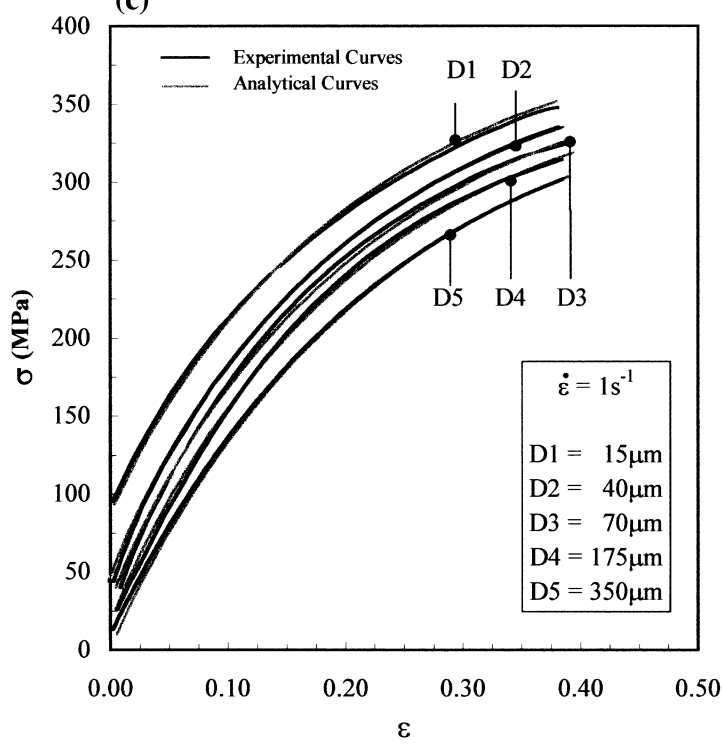

$$
\frac{x}{k}=\frac{k_{2}}{k_{1}^{2}}=\frac{c b \beta^{2}}{D} .
$$

In Fig. 9 the evolution of $x / k$ values as a function of the grain size is shown. The relationship between $x / k$ and $(1 / D)$ corroborates the ability of the geometrical necessary dislocation (as proposed by Ashby) to take into account the grain size in polycrystalline copper, in the range of grain sizes considered in this work. The slope of the linear fitting to the mean experimental points is in agreement with the values indicated in the literature for $c$ and $\beta$.

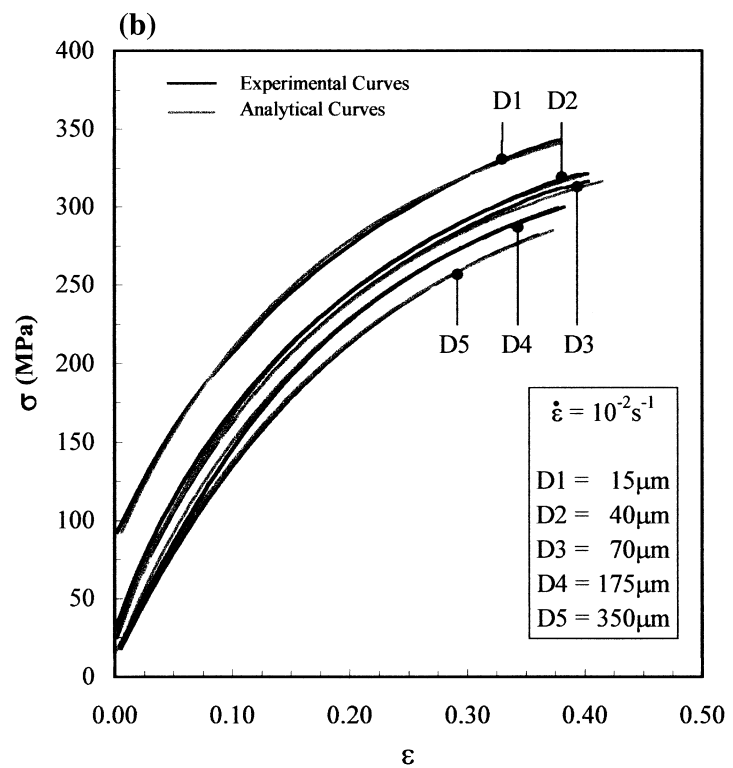

Fig. 7. Examples of fitting of the experimental results for all the grain sizes studied $(15,40,70,175$ and $350 \mu \mathrm{m}$ ), by using equation (24) for: (a) $\dot{\varepsilon}=10^{-4} / \mathrm{s}$; (b) $\dot{\varepsilon}=10^{-2} / \mathrm{s}$; and (c) $\dot{\varepsilon}=1 / \mathrm{s}$. 


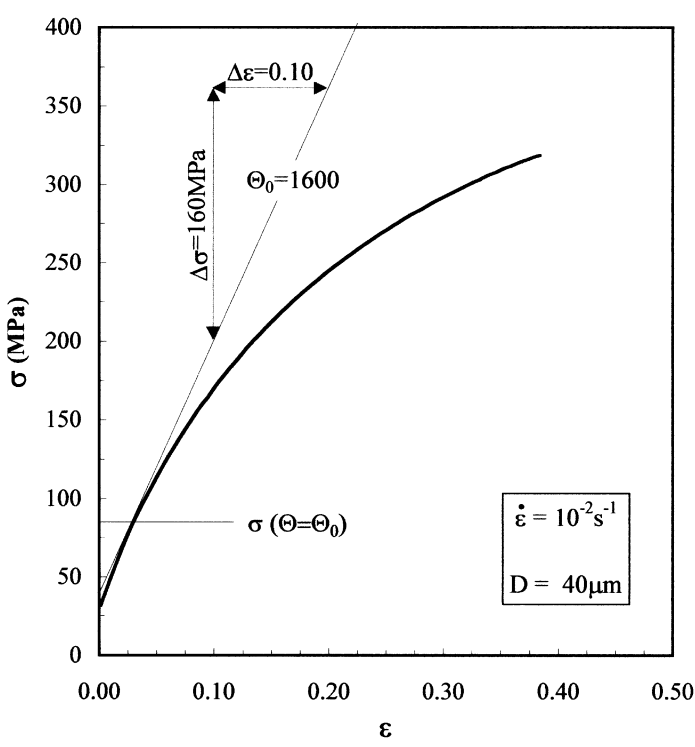

Fig. 8. An expedite method to determine the value of $\sigma\left(\Theta=\Theta_{0}\right)$, directly on the true stress $(\sigma)$-true strain $(\varepsilon)$ curves.

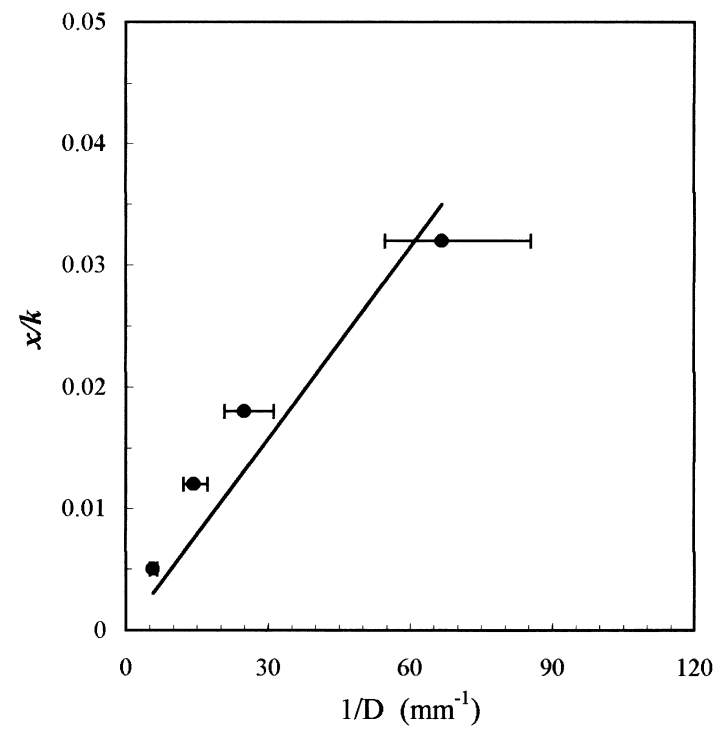

Fig. 9. Evolution of $x / k$ values as a function of the inverse of the grain size.

\section{CONCLUSION}

Further development of the hybrid model was carried out in order to get a better understanding of the relative influence of statistically stored $\rho^{\mathrm{S}}$ and geometrical $\rho^{\mathrm{G}}$ dislocations (introduced by Ashby) on the stress-strain curves. The evolution of the ratio between the cumulated production of both types of dislocation densities with deformation $\gamma$ is as follows:

$$
\begin{aligned}
\log \left(\frac{\rho^{\mathrm{G}}}{\rho^{\mathrm{S}}}\right) & =-\log \gamma+\log \left(4 k_{2} / k_{1}^{2}\right) \\
& =-\log \gamma+\log (4 x / k)
\end{aligned}
$$

where

$$
x=\frac{k k_{2}}{k_{1}^{2}}
$$

The values $k_{1}$ and $k_{2}$ define the rate of dislocation production $\rho^{\mathrm{S}}$ and $\rho^{\mathrm{G}}$, respectively, and $k$ is a value that defines the rate of dislocation annihilation at each temperature, strain rate and grain size. The parameter $x$ relates the values of $k, k_{1}$ and $k_{2}$, the definition of which is made by the equation that characterises the evolution of the total dislocation density $\rho$ with deformation $\gamma$ as follows:

$$
\frac{\mathrm{d} \rho}{\mathrm{d} \gamma}=k_{1} \sqrt{\rho}+k_{2}-k \rho .
$$

By integrating the above equation, it was possible to conclude that the geometrical dislocation density shows its importance long after the respective density becomes much less than the statistical dislocation.

The analysis performed leads to an equation which is able to describe the work-hardening behaviour of large and small grain size samples:

$$
\frac{\Theta}{\Theta_{0}}=1+\frac{x}{\frac{\sigma}{\sigma_{\text {sat }}^{\mathrm{V}}}} \frac{k^{\mathrm{V}}}{k}-\frac{\sigma}{\sigma_{\text {sat }}^{\mathrm{V}}} \frac{k}{k^{\mathrm{V}}}
$$

where $\Theta=\mathrm{d} \sigma / \mathrm{d} \varepsilon$ ( $\sigma$ and $\varepsilon$ are the macroscopic stress and strain, respectively), $\Theta_{0}$ is the athermal strain hardening rate related to stage II of monocrystal deformation, and $\sigma_{\text {sat }}^{\mathrm{V}}$ and $k^{\mathrm{V}}$ concern the case without geometrical dislocations (i.e. monocrystal or large grain size polycrystal).

The analytical description of the resulting flow law was used to analyse the tensile behaviour of copper sheets with four different grain sizes deformed at three different strain rates. It could be concluded that the value of $x$ is dependent on the grain size, and almost does not depend on the strain rate.

\section{REFERENCES}

1. Sevillano, J. G., van Houtte, P. and Aernoudt, E., Prog. Mater. Sci., 1980, 25, 69.

2. Kuhlmann-Wilsdorf, D., Mater. Sci. Eng., 1989, A113, 1.

3. Bay, B., Hansen, N., Hughes, D. A. and KuhlmannWilsdorf, D., Acta metall. mater., 1992, 40, 205.

4. Hansen, N., in Proc. 15th Riso Int. Symposium on Metall. and Mater. Sci.: Numerical Predictions of Deformation Processes and the Behaviour of Real Materials, ed. S. I. Andersen, J. B. Bilde-Sørensen, T. Lorentzen, O. B. Pedersen and N. J. Sørensen. Risø National Laboratory Roskilde, 1994, pp. 325-334.

5. Nes, E., Prog Mater. Sci., 1998, 41, 129. 
6. Kocks, U. F., J. Engng. Mater. Technol. (Trans. ASME), 1976, 98, 76.

7. Mecking, H. and Kocks, U. F., Acta metall., 1981, 29, 1865.

8. Estrin, Y. and Mecking, H., Acta metall., 1984, 32, 57.

9. Mecking, H. and Estrin, Y., in Proc. 8th Riso Int. Symposium on Metall. and Mater. Sci.: Constitutive Relationships and their Physical Basis, ed. S. I. Andersen, J. B. Bilde-Sørensen, N. Hansen, T. Leffers, H. Lilholt, O. B. Pedersen and B. Ralph. Risø National Laboratory, Roskilde, 1987, pp. 123145.

10. Estrin, Y., in Unified Constitutive Laws of Plastic Deformation, ed. A. S. Krausz and K. Krausz. Academic Press, 1995.

11. Voce, E., J. Inst. Metals, 1948, 74, 537.

12. Voce, E., Metallurgia, 1955, 51, 219.

13. Taylor, G. I., J. Inst. Metals, 1938, 62, 307.

14. Kocks, U. F., Metall. Trans., 1970, 1, 1121.

15. Tome, C., Canova, G. R., Kocks, U. F., Christodoulou, N. and Jonas, J. J., Acta metall., 1984, 32, 1637.

16. Thompson, A. W., Metall. Trans., 1977, 8A, 833.

17. Hansen, N., Acta metall., 1977, 25, 863.

18. Hansen, N. and Ralph, B., Acta metall., 1982, 30, 411.

19. Hansen, N., Metall. Trans., 1985, 16A, 2167.

20. Shankaranarayan, H. and Varma, S. A., J. Mater. Sci., 1995, 30, 3576

21. Margolin, H. and Stanesco, M. S., Acta metall., 1975, 23, 1411 .
22. Meyers, M. A., Andrade, U. R. and Chokshi, A. H., Metall. Trans., 1995, 26A, 2881.

23. Jiang, Z., Lian, J. and Baudelet, B., Acta metall. mater., 1995, 43, 3349.

24. Gracio, J. J., Fernandes, J. V. and Schmitt, J. H., Mat. Sci. Eng., 1989, A118, 97.

25. Ashby, M. F., Phil. Mag., 1970, 21, 399.

26. Essmann, U. and Mughrabi, H., Phil. Mag., 1979, 40, 731.

27. Prinz, F. B. and Argon, A. S., Acta metall., 1984, 32, 1021.

28. Nix, W. D., Gibeling, J. C. and Hughes, D. A., Metall. Trans., 1985, 16A, 2215.

29. Sevillano, J. G., in Materials Science and Technology: A Comprehensive Treatment, Vol. 6, ed. R. W. Cahn, P. Haasen, E. J. Kramer. Plastic Deformation and Fracture of Metals, ed. H. Mughrabi. VCH, Weinheim, 1993, p. 19-88.

30. Aernoudt, E., Sevillano, J. G. and van Houtte, P., in Proc. 8th Riso Int. Symposium on Metall. and Mater. Sci.: Constitutive Relationships and their Physical Basis, ed. S. I. Andersen, J. B. Bilde-Sørensen, N. Hansen, T. Leffers, H. Lilholt, O. B. Pedersen and B. Ralph. Risø National Laboratory, Roskilde, 1987, pp. $1-38$.

31. Thompson, A. W. and Baskes, M. I., Phil. Mag., 1973, 28, 301.

32. Thompson, A. W., Metall. Trans., 1974, 5, 39.

33. Nes, E., Acta metall. mater., 1995, 43, 2189.

34. Furu, T. Ørsund R. and Nes, E., Acta metall. mater., 1995, 43, 2209 